\title{
PRINCIPAIS INTERCORRÊNCIAS E DESFECHOS CLÍNICOS DE IDOSOS VÍTIMAS DE TRAUMA NA UNIDADE DE TERAPIA INTENSIVA*
}

\author{
Ana Cristina Carvalho da Costa ${ }^{1}$, Renata Costa Fortes ${ }^{2}$
}

Objetivo: Identificar as principais intercorrências e o desfecho clínico de idosos internados por causas traumáticas na Unidade de Terapia Intensiva de um hospital de referência do Distrito Federal admitidos entre julho de 2012 e julho de 2014. Método: Estudo retrospectivo analítico, com coleta de dados em prontuário. Os dados foram analisados com os testes Qui-Quadrado e Mann-Whitney, considerando como significativo um valor de $p<0,05$. Resultados: Dentre 91 idosos, houve prevalência do sexo masculino e da queda da própria estatura, como mecanismo de lesão. As principais intercorrências foram: infecção pulmonar, traqueostomia decorrente de intubação prolongada e choque séptico. Observou-se alta mortalidade, $38(41,7 \%)$, associada ao sexo masculino $(p=0,029)$, às outras causas externas $(p=0,03)$, ao choque séptico $(p=0,039)$ e à diálise $(p=0,015)$. Conclusão: A maior gravidade apresentada favoreceu a ocorrência de múltiplas intercorrências durante a internação na terapia intensiva; entretanto, apesar do pior prognóstico, os idosos podem sobreviver ao trauma e suas complicações associadas.

DESCRITORES: Idoso; Causas externas; Ferimentos e lesões; Mortalidade; Unidade de terapia intensiva.

\section{MAIN COMPLICATIONS AND CLINICAL OUTCOMES OF ELDERLY TRAUMA VICTIMS ADMITTED TO INTENSIVE THERAPY UNIT}

Objective: To identify the main complications and the clinical outcome of elderly trauma victims admitted to the Intensive Care Unit of a referral hospital of Distrito Federal between July 2012 and July2014. Method: Retrospective analytical study that uses medical records. Data were analyzed using Chi-Square and Mann-Whitney tests, and a significance value of $p<0.05$ was considered. Results: Most of the 91 elderly were men, whose mechanism of injury was falls from standing height. The main complications were pulmonary infection, tracheostomy due to prolonged intubation and septic shock. There was a high mortality rate: $38(41.7 \%)$, associated with male gender $(p=0.029)$, other external causes $(p=0.03)$, septic shock $(p=0.039)$ and dialysis $(p=0.015)$. Conclusion: The severe condition of the patients favored the occurrence of multiple complications during hospitalization in intensive care. However, despite the worst prognosis, the elderly may survive the trauma and its associated complications.

KEYWORDS: Elderly; External causes; Wounds and injuries; Mortality: Intensive care unit

\section{PRINCIPALES COMPLICACIONES Y SUS RESULTADOS CLÍNICOS DE ANCIANOS VÍCTIMAS DE TRAUMA EN LA UNIDAD DE TERAPIA INTENSIVA}

\begin{abstract}
Objetivo: Identificar las principales complicaciones y su resultado clínico asociadas a ancianos ingresados a causa de traumas en la Unidad de Terapia Intensiva de un hospital de referencia de Distrito Federal entre julio de 2012 y julio de 2014. Método: Estudio retrospectivo analítico, con obtención de datos en prontuario. Se analizaron los datos por medios de los test Chi cuadrado y Mann-Whitney, considerando como significativo un valor de p<0,05. Resultados: De 91 ancianos, hubo prevalencia del sexo masculino y de la caída de la propia estatura, como mecanismo de lesión. Las principales complicaciones fueron infección pulmonar, traqueostomía proveniente de intubación prolongada y choque séptico. Se observó gran mortalidad, $38(41,7 \%)$, asociada al sexo masculino $(p=0,029)$, a otras causas externas $(p=0,03)$, al choque séptico $(p=0,039)$ y a la diálisis $(p=0,015)$. Conclusión: La mayor gravedad presentada favoreció la ocurrencia de múltiples complicaciones durante el ingreso en la terapia intensiva; sin embargo, a pesar del peor pronóstico, los ancianos pueden sobrevivir al trauma y sus complicaciones asociadas.
\end{abstract}

DESCRIPTORES: Anciano; Causas externas; Herimientos y lesiones; Mortalidad; Unidad de terapia intensiva.

\footnotetext{
"Artigo extraído da dissertação intitulada: "Idosos vítimas de trauma internados na unidade de terapia intensiva e relação entre idade, comorbidades, complicações e desfechos clínicos: um estudo retrospectivo analítico". Fundação de Ensino e Pesquisa em Ciências da Saúde, 2015.

${ }^{1}$ Enfermeira. Mestre em Ciências da Saúde. Enfermeira assistencial da Unidade de Terapia Intensiva Adulto da Secretaria de Estado de Saúde do Distrito Federal. Brasília, DF, Brasil.

${ }^{2}$ Nutricionista. Doutora em Nutrição Humana. Docente do Programa de Mestrado Profissional em Ciências da Saúde da Fundação de Ensino e Pesquisa em Ciências da Saúde. Brasília, DF, Brasil.
}

\section{Autor Correspondente:}

Ana Cristina Carvalho da Costa.

Secretaria de Estado de Saúde do Distrito Federal.

Rua 36 Norte, 3350, D, 102 - 71919-180. Brasília, DF, Brasil.

E-mail: anacristina_costa@yahoo.com.br
Recebido: 21/09/2017

Finalizado: 16/04/2018 


\section{INTRODUÇÃO}

O envelhecimento populacional caracteriza-se pela redução da participação relativa de crianças e jovens, acompanhada do aumento proporcional dos adultos e, particularmente, dos idosos em uma sociedade. No Brasil, a população de idosos, indivíduos com 60 anos ou mais de idade, representa, aproximadamente, $10,8 \%$ dos residentes no país, o que corresponde a mais de vinte milhões de indivíduos, com predominância das mulheres (55,5\%). Tem-se um aumento na expectativa de vida em todas as idades, inclusive entre os idosos. ${ }^{(1)}$

Paralelamente ao aumento significativo do número de idosos, devido à elevação da expectativa de vida, ao decréscimo nas taxas de mortalidade e natalidade, e à busca por um modo de vida mais ativo, o número de traumas vem aumentando nessa população. Este fenômeno é descrito como lesões advindas de força, podendo ser de natureza acidental, autoimposta ou um ato de violência, que atinge um ou mais sistemas e requer atendimento imediato. ${ }^{(2)}$

As causas traumáticas são consideradas importantes determinantes de morbimortalidade em idosos, representadas basicamente pelas quedas e acidentes de trânsito. ${ }^{(1)} \mathrm{O}$ trauma em idosos possui características específicas e alguns fatores, como as alterações decorrentes do próprio processo de envelhecimento, a associação a doenças preexistentes e o uso concomitante de medicamentos, podem interferir negativamente em sua morbimortalidade. ${ }^{(3)}$

Os eventos traumáticos são uma das principais causas de lesões, incapacidades e internações em lares e outras instituições, constituindo a quinta causa de mortalidade entre os indivíduos com mais de 75 anos de idade. Os idosos vítimas de trauma apresentam-se inicialmente de modo mais crítico, necessitam de internação hospitalar com maior frequência, representam grande proporção dos pacientes internados em Unidade de Tratamento Intensivo (UTI) e consomem mais recursos do que os pacientes de qualquer outro grupo etário. ${ }^{(3)}$

Considerando o expressivo aumento da população idosa, os custos associados e a mortalidade devido ao trauma no idoso, ainda há poucos estudos que tratam do idoso vítima de trauma internado no ambiente de alta complexidade, cenário de internação de pacientes gravemente enfermos, com emprego de modernas tecnologias, equipe especializada e geração de altos custos para o Sistema Único de Saúde (SUS).

Assim, esse tema surge como de relevância na saúde pública, uma vez que alternativas que minimizem as complicações e a morbimortalidade associadas ao trauma podem ser identificadas e validadas para uso na assistência prestada a esses pacientes, trazendo melhorias aos processos de trabalho, otimizando os recursos existentes, agregando qualidade ao cuidado prestado e reduzindo custos associados à internação ao SUS.

O objetivo deste estudo foi identificar as principais intercorrências e o desfecho clínico de idosos internados por causas traumáticas na Unidade de Terapia Intensiva de um hospital de referência.

\section{MÉTODO}

Estudo retrospectivo analítico, com abordagem quantitativa, cujos dados foram coletados na UTI de um hospital de referência para atendimento a vítimas de trauma, da Secretaria de Estado de Saúde do Distrito Federal (SES-DF), localizado em Brasília, Distrito Federal.

Foram incluídos no estudo pacientes com idade igual ou superior a 60 anos internados por causas traumáticas na UTI, no período compreendido entre julho de 2012 e julho de 2014. A amostra foi determinada pelo critério de amostragem não-probabilística por conveniência. Foram excluídos os pacientes idosos admitidos por causas clínicas ou cirúrgicas não traumáticas e aqueles cujas informações nos prontuários sobre os mecanismos e causas traumáticas estavam incompletas.

A pesquisa foi realizada na base eletrônica de dados da SES-DF, o sistema de prontuário informatizado TRACKCARE $^{\circledR}$, sendo coletadas informações sócio demográficas (sexo, raça, idade, peso, estatura, 
escolaridade), aquelas relacionadas ao mecanismo do trauma, às comorbidades associadas e à internação na UTI (tempo de internação, uso de dispositivos invasivos, drogas vasoativas e sedativos, complicações e desfechos).

Pela importância reconhecida das quedas na epidemiologia do trauma na população idosa, e apesar de ser considerada uma causa externa pelo Código Internacional de Doenças (CID-10), decidiu-se analisar separadamente os dados relativos aos idosos vítimas de trauma por queda e por outras causas externas (acidentes e violência). Assim, foi possível avaliar de maneira mais sensível o impacto de cada mecanismo causal no estado de saúde do idoso vítima de trauma internado na UTI.

Para análise dos dados, utilizou-se a estatística descritiva, tabelas de frequência absoluta e percentual. Os dados foram registrados em planilha eletrônica do software Microsoft Excel (versão 14.0) e para a análise das associações foi utilizado o teste do Qui-Quadrado e o teste de Mann-Whitney para variáveis não paramétricas, por meio do software Statistical Package and Service Solutions (SPSS) versão 18.0. O teste de normalidade utilizado foi o teste de Shapiro-Wilk. A probabilidade de significância estatística aceitável foi de $p<0,05$.

Esta pesquisa teve aprovação pelo Comitê de Ética em Pesquisa da Fundação de Ensino e Pesquisa em Ciências da Saúde da Secretaria de Estado de Saúde do Distrito Federal (CEP/FEPECS/SES-DF), sob os números de parecer 453.470 e 882.249.

\section{RESUltADOS}

Entre julho de 2012 e julho de 2014, 2652 pacientes foram admitidos na UTI do referido hospital de estudo; destes, 960 (36,2\%) eram idosos. Na população estudada foram selecionados 103 (10,7\%) que correspondiam aos idosos admitidos por causas traumáticas. Destes, 12 (11,6\%) pacientes foram excluídos da amostra por apresentarem prontuários com dados incompletos sobre o mecanismo e causa traumática. Assim, a amostra final foi composta por 91 idosos vítimas de trauma, o que correspondeu a 9,5\% dos idosos admitidos ou 3,4\% do total de admissões nesse período.

Em relação ao sexo, observou-se predominância do sexo masculino em relação ao feminino, numa proporção de 2,6:1. Quanto à faixa etária, constatou-se média de idade de 72 7,8 anos (de 60 a 92 anos), sendo superior no sexo feminino (76 $\pm 7,1$ anos) quando comparada ao masculino $(70,4 \pm 7,6$ anos). Entretanto, não houve diferença significativa entre os sexos $(p=0,19)$. Houve predomínio de idosos na faixa etária de 60 a 69 anos $(42,8 \%)$, seguidos de 70 a 79 anos (38,5\%). (Tabela 1).

Tabela 1 - Distribuição dos idosos vítimas de trauma internados na Unidade de Terapia Intensiva, por faixa etária e sexo. Brasília, DF, Brasil, 2015

\begin{tabular}{lccccc}
\hline \multicolumn{1}{c}{ Idade } & $\begin{array}{c}\mathbf{6 0 - 6 9} \text { anos } \\
\mathbf{n ( \% )}\end{array}$ & $\begin{array}{c}\mathbf{7 0 - 7 9} \text { anos } \\
\mathbf{n}(\%)\end{array}$ & $\begin{array}{c}\mathbf{8 0 - 8 9} \text { anos } \\
\mathbf{n}(\%)\end{array}$ & $\begin{array}{c}>\mathbf{9 0} \text { anos } \\
\mathbf{n}(\%)\end{array}$ & $\begin{array}{c}\text { Total } \\
\mathbf{n}(\%)\end{array}$ \\
\hline Feminino & $4(4,3)$ & $14(15,4)$ & $5(5,5)$ & $2(2,2)$ & $25(27,5)$ \\
Masculino & $35(38,5)$ & $21(23,1)$ & $9(9,9)$ & $1(1,1)$ & $66(72,5)$ \\
\hline Total & $39(42,8)$ & $35(38,5)$ & $14(15,4)$ & $3(3,3)$ & $91(100)$
\end{tabular}

Ao analisarmos as doenças preexistentes, constatou-se que $60(65,9 \%)$ idosos apresentavam alguma comorbidade; destacando-se a hipertensão arterial $(n=36,60 \%)$, o etilismo $(n=13,21,7 \%)$, o diabetes mellitus tipo $2(n=11,18,3 \%)$ e o acidente vascular encefálico do tipo isquêmico $(n=10,16,7 \%)$. A média de doenças preexistentes por idoso foi de 2,4, sendo que em 31 prontuários $(34,1 \%)$ havia a informação de ausência de doenças pregressas ou falta de registro sobre as mesmas. 
Houve associação entre sexo e comorbidades apresentadas; nas pacientes do sexo feminino houve maior ocorrência de hipertensão $(p=0,017)$ e diabetes $(p=0,041)$, enquanto que no sexo masculino o acidente vascular encefálico isquêmico $(p=0,026)$ e o etilismo $(p=0,001)$ foram mais frequentes.

Não foi possível encontrar registros sobre raça, escolaridade e estado civil, uma vez que esses dados estavam ausentes em $80(87,9 \%)$ prontuários pesquisados. Quanto ao atendimento pré-hospitalar, 51 $(56 \%)$ idosos receberam esse tipo de suporte, sendo atendidos pelo Serviço de Atendimento Móvel de Urgência (SAMU), o qual foi responsável por $42(82,4 \%)$ atendimentos, em especial às vítimas de outras causas externas $(n=26,61,9 \%)$. De um total de 52 idosos vítimas de queda, 50\% dos casos (n=26) não buscou atendimento pré-hospitalar, dirigindo-se do próprio domicílio ao serviço de saúde de emergência, com destaque para os que sofreram queda da própria estatura ( $n=20,76,9 \%)$.

Em relação ao mecanismo de lesão, houve predomínio da queda ( $n=52,57,1 \%$ ), em especial as quedas da própria estatura $(n=38,41,7 \%)$, quando comparado às outras causas externas (acidentes e violência) presentes em 39 casos (42,9\%), com destaque para os atropelamentos $(n=15,16,5 \%)$. Quanto às principais causas traumáticas de admissão, houve predomínio do traumatismo crânio-encefálico $(n=51,56 \%)$, seguido do politrauma $(n=21,23,1 \%)$ e das fraturas $(n=16,17,6 \%)$. (Tabela 2$)$.

Tabela 2 - Causas traumáticas de admissão e mecanismos de lesão dos idosos vítimas de trauma internados na Unidade de Terapia Intensiva. Brasília, DF, Brasil, 2015

\begin{tabular}{lccc} 
Causas traumáticas & $\begin{array}{c}\text { Queda } \\
\mathbf{n}(\%)\end{array}$ & $\begin{array}{c}\text { Outras Causas } \\
\text { Externas } \\
\mathbf{n}(\%)\end{array}$ & Total \\
\hline $\begin{array}{l}\text { Traumatismo crânio-encefálico } \\
\text { Leve }\end{array}$ & $3(3,3)$ & $1(1,1)$ & $51(56)$ \\
$\quad \begin{array}{l}\text { Moderado } \\
\text { Grave }\end{array}$ & $4(4,4)$ & $2(2,2)$ & \\
Politrauma & $28(30,8)$ & $13(14,3)$ & $21(23,1)$ \\
Traumatismo raquimedular & $1(1,1$ & $20(22)$ & $3(3,3)$ \\
$\quad$ Cervical & & $1(1,1)$ & \\
$\quad$ Torácico & 0 & $1(1,1)$ & $16(17,6)$ \\
$\quad$ Lombar & 0 & 0 & \\
$\quad$ Quaturas & $1(1,1)$ & 0 & $91(100)$ \\
$\quad$ Fêmur & $2(2,2)$ & $1(1,1)$ & \\
\hline Total & $13(14,3)$ & $39(42,9)$ & \\
\hline
\end{tabular}

A média de tempo de espera por um leito de UTI entre os idosos da amostra foi de 55,8 horas (1h-312h) ou 2,35 dias. Já a média de tempo de internação na terapia intensiva foi de 346,9 horas (12h-2544h), ou 14,45 dias.

Quanto aos dispositivos invasivos, a média de dispositivos utilizados foi de cinco (1-9). Já em relação ao uso de drogas vasoativas e sedativos, $67(73,6 \%)$ idosos utilizaram drogas vasoativas durante a internação, em especial noradrenalina $(n=63,69,2 \%)$ e nitroprussiato de sódio $(n=11,12,1 \%) ; 65$ pacientes $(71,4 \%)$ utilizaram sedativos, com destaque para o fentanil $(n=58,63,7 \%)$, o midazolam $(n=53,58,2 \%)$ e o propofol $(n=36,39,6 \%)$.

Dentre as intercorrências apresentadas durante a internação na UTI, destacaram-se a infecção de foco pulmonar $(n=41,45 \%)$, a realização de traqueostomia após intubação traqueal prolongada ou falha de extubação $(n=38,41,7 \%)$, o choque séptico $(n=31,34,1 \%)$, o uso de hemoderivados $(n=30$, $33 \%)$ e a insuficiência renal aguda $(n=26,28,6 \%)$, sendo que $19,8 \%(n=18)$ necessitaram de terapia de substituição renal (hemodiálise). Ressalta-se que 67 (73,6\%) apresentaram mais de uma complicação durante a internação e que apenas nove $(9,9 \%)$ idosos não apresentaram complicações. 
Já em relação ao desfecho clínico do idoso na UTI, 38 (41,8\%) faleceram, 47 (51,6\%) foram de alta para a enfermaria e seis $(6,6 \%)$ foram transferidos para unidades de terapia intensiva de menor complexidade, por resolução da causa traumática com permanência da dependência da ventilação mecânica. Entre os idosos que evoluíram ao óbito, houve predomínio do sexo masculino (n=29, 76,3\%) e das vítimas de outras causas externas ( $n=20,52,6 \%$ ).

\section{DISCUSSÃO}

Dentre os pacientes admitidos na UTI, os idosos correspondiam a mais de $30 \%$ do total de pacientes internados, sendo que os idosos vítimas de trauma representavam cerca de $10 \%$ dessa população. Situação semelhante à encontrada em diversos estudos, em que a prevalência de trauma entre os jovens é mais acentuada. Na população geriátrica, a maior prevalência é de emergências clínicas; entretanto, o número de idosos traumatizados vem aumentando, o que se configura como um evento relevante, uma vez que lesões que poderiam ser facilmente toleradas por pacientes mais jovens podem resultar em taxas mais altas de mortalidade nos idosos. ${ }^{(4-6)}$

Em relação ao sexo, os dados obtidos neste estudo vão de encontro ao que foi observado nos artigos consultados, em que o sexo feminino foi predominante. Já em relação à idade, houve concordância com a literatura. ${ }^{(4-5,7-8)}$

Supõe-se que o predomínio do sexo masculino ( $n=66,72,5 \%)$ esteja ligado à média de idade menor, como a apresentada pelos idosos do estudo. Idosos na faixa etária de 60 a 69 anos, a mais prevalente no presente estudo, geralmente ainda apresenta perfil de população adulta, ou seja, com independência e autonomia, o que lhes proporciona vida ativa com participação em atividades sociais, de trabalho, de lazer, que podem expô-los a situações de trauma. ${ }^{(3)}$

Provavelmente, homens idosos nas faixas etárias mais novas se exponham mais a traumas pelo fato de que circulam mais pelo perímetro urbano e estão mais inseridos no mercado de trabalho; enquanto que as idosas circulam, geralmente, por lugares mais próximos de seus domicílios. Ademais, as práticas de risco estão fortemente associadas ao sexo masculino, o que torna os homens as maiores vítimas da violência, potencializada por desigualdades sociais e outras condições adversas.(2)

Neste estudo foi observada a predominância de idosos ( $n=60,65,9 \%$ ) com doenças crônicas preexistentes, destacando-se a hipertensão arterial sistêmica. Acima de 50\% da população geriátrica vítima de trauma tem hipertensão arterial não diagnosticada, e mais de $30 \%$ possui doença cardíaca. Diabetes, eventos cerebrovasculares prévios, doença pulmonar obstrutiva crônica (DPOC), demência, arritmias e distúrbios endócrinos estão presentes em cerca de 10\% dessa população. Outras doenças comuns entre os idosos, como infecções respiratórias, neoplasias e insuficiência renal crônica, também aumentam o risco de complicações após o trauma. ${ }^{(9)}$

Ressalta-se que o etilismo configurou-se como segundo lugar dentre as comorbidades relatadas pelos idosos deste estudo, presente em 14,3\% dos casos. Estudo realizado na região nordeste do Brasil encontrou dado semelhante e inferiu que o uso de bebidas alcoólicas constitui um dos principais fatores que colaboram para a elevação das taxas de mortalidade por causas externas. São diversas as justificativas que levam ao uso do álcool, entre elas, o fácil acesso a esse produto, o baixo custo e o estímulo ao consumo pelos meios de publicidade. ${ }^{(2)}$

Quanto às principais causas traumáticas de admissão, foram encontrados resultados semelhantes a outros estudos, com predomínio da queda $(n=52,57,1 \%)$, principalmente da própria estatura (41,7\%). A queda, de acordo com a $10^{a}$ revisão da Classificação Internacional de Doenças (CID-10), é considerada uma causa externa. É definida como "um evento acidental decorrente da mudança de posição do indivíduo para um nível mais baixo em relação à posição que se encontrava inicialmente", podendo ser classificada em queda de mesmo nível ou de nível elevado. ${ }^{(10)}$

A queda de mesmo nível, mais prevalente no presente estudo, é definida como evento não intencional que leva uma pessoa inadvertidamente a cair ao chão em um mesmo nível ou em outro inferior. É considerada problema de saúde pública e está associada a fatores intrínsecos e extrínsecos. As 
complicações decorrentes da queda no idoso podem ser caracterizadas pela imobilização prolongada, rabdomiólise, desidratação e infecção. Além disso, as lesões decorrentes de quedas em pacientes geriátricos tendem a ser mais graves do que aquelas apresentadas por pacientes jovens e com quedas similares. Estudos mostram que 30\% das pessoas com idade superior a 65 anos caem pelo menos uma vez ao ano e que metade dessas pessoas experimentam outras quedas, após o primeiro evento. ${ }^{(11-13)}$

As quedas, em especial as da própria estatura, foram os principais mecanismos de lesão entre idosos em diversas pesquisas, seguidos por acidentes de trânsito e violência interpessoal. ${ }^{(11-13)}$ No presente estudo, outras causas externas (acidentes e violências) representaram 42,9\% dos casos, com destaque para os atropelamentos com ocorrência de 16,5\%. Apesar de não ter sido a causa traumática mais prevalente, outras causas externas estiveram presentes em 52,6\% dos óbitos, mostrando sua importância nesse grupo etário. Esses dados estão em consonância com a literatura, em que o atropelamento corresponde à principal causa de morte para idosos dentre os acidentes de trânsito. ${ }^{(1)}$

O processo de envelhecimento leva gradualmente à diminuição dos reflexos, da agilidade e da força, associado à maior prevalência de doenças crônicas em idosos e ao uso de polifarmácia para tratamento das comorbidades, levando a um maior risco para eventos traumáticos nessa faixa etária. ${ }^{(14)}$

Quanto à presença de atendimento pré-hospitalar, 51 pacientes (56\%) receberam esse tipo de suporte. Entretanto, nesse estudo, houve associação significativa entre a ausência de atendimento pré-hospitalar e a mortalidade $(p=0,037)$, em especial no caso dos idosos vítimas de outras causas externas, os quais apresentaram lesões em maior número e maior gravidade. A contribuição do atendimento pré-hospitalar móvel para redução da mortalidade e das sequelas nas vítimas de trauma é inegável, pois evita um primeiro atendimento tardio ou inadequado. ${ }^{(3)}$

No presente estudo, houve predomínio do trauma crânio-encefálico, principalmente do tipo grave, seguido do politrauma e das fraturas. Há evidências de que as lesões de extremidades são mais frequentes, porém, que as lesões presentes no segmento cefálico são mais graves, podendo acarretar hemorragias intracranianas e contusões cerebrais, levando à maior probabilidade de óbito. A literatura traz que os politraumatismos entre os idosos são casos incomuns; entretanto, 21 pacientes do estudo sofreram esse tipo de lesão, demonstrando a maior gravidade de seus casos. ${ }^{(14-16)}$

O uso de grande número de dispositivos invasivos para a manutenção do paciente grave na UTI o predispõe a maior risco de infecção. O uso de drogas vasoativas e sedativos em mais de $70 \%$ dos pacientes evidencia a gravidade do quadro clínico dos mesmos, necessitando de medidas rigorosas de neuroproteção e de suporte hemodinâmico.

Deve-se considerar que no caso do idoso vítima de trauma gravemente enfermo podem ocorrer alterações cardíacas e/ou renais, demandando atenção especial no controle da diurese e o exame frequente dos aparelhos cardiovascular e respiratório do idoso, com vistas à prevenção de complicações. É sugerido que a abordagem inicial mais agressiva neste tipo de paciente esteja relacionada à maior taxa de sobrevida. Dentre os procedimentos propostos estão a monitorização hemodinâmica invasiva, a hemodiálise e o suporte nutricional precoce. ${ }^{(3,9)}$

O tempo médio entre a indicação de internação na terapia intensiva e a admissão do paciente na UTI, encontrado no presente estudo, foi de 55,8 horas, ou seja, um pouco maior que dois dias. Estudo brasileiro $^{(17)}$ aponta recomendações para a admissão na unidade de cuidados intensivos em até 72 horas; entretanto, pesquisa americana ${ }^{(18)}$ recomenda a admissão em tempo menor, de no máximo seis (6) horas. De acordo com esses artigos, ${ }^{(17-18)}$ o tempo de espera prolongado por um leito de UTI pode agravar o quadro clínico, aumentar o tempo de internação e elevar o risco de mortalidade, de forma independente, em cerca de $1,5 \%$ a cada hora de espera.

Muitos estudos abordam somente o tempo de internação no setor de emergência ou o tempo total de internação hospitalar dos idosos vítimas de trauma, com média de seis dias de internação, sendo de até dez dias para traumas de alta energia. ${ }^{(14)}$ Neste estudo, a média de tempo de internação somente na UTI foi de 14 dias, o que mais uma vez corrobora a gravidade dos pacientes internados e o alto custo associado ao trauma nessa população. O tempo de internação prolongado é um fator preocupante quando se trata de pacientes idosos, considerando a possibilidade de complicações e principalmente o declínio funcional aos quais esses se tornam vulneráveis. ${ }^{(7)}$ 
O período pós-trauma é considerado crítico, pois o idoso torna-se ainda mais susceptível ao comprometimento da função cardiopulmonar, ao aparecimento da trombose venosa profunda, à atrofia muscular, às alterações articulares e às lesões por pressão. Daí supõe-se que os idosos traumatizados estão mais propensos a ter complicações durante a internação na UTI. ${ }^{(11)}$ Foram encontradas como principais complicações apresentadas pelos idosos do estudo a infecção pulmonar; o choque séptico; a realização de traqueostomia, devido à intubação prolongada ou à falha de extubação; o uso de hemoderivados; e a insuficiência renal aguda, em especial com necessidade de suporte dialítico.

Estudos demonstram que em pacientes vítimas de trauma internados em UTI, a idade é um preditor independente de mortalidade. Além disso, doença renal preexistente e necessidade de terapia de substituição renal durante a internação também são preditores de mortalidade. Pacientes com trauma grave, que apresentaram disfunção orgânica e uso de drogas inotrópicas, assim como comprometimento cardíaco, diálise e ventilação mecânica apresentaram maior mortalidade e pior sobrevida após a alta da UTI. ${ }^{(15,19-20)}$

A mortalidade nos pacientes estudados foi de $41,8 \%$ ( $n=38)$, com maior prevalência no sexo masculino e nas vítimas de outras causas externas. Dado alarmante e que vai de encontro ao relatado na literatura, em que a mortalidade variou de $7,4 \%$ a $12,2 \%$, embora alguns estudos relatem que pacientes que necessitaram de diálise tiveram mortalidade de $33 \% .{ }^{(14,19,21)}$ No presente estudo, não houve associação significativa entre idade e mortalidade $(p=0,42)$; entretanto, houve associação significativa entre a mortalidade e o sexo masculino $(p=0,029)$, a ocorrência de outras causas externas $(p=0,03)$, o choque séptico de foco pulmonar ( $p=0,039)$ e a diálise $(p=0,015)$.

Diante da alta mortalidade encontrada, estudos apontam que os coeficientes de mortalidade pelas causas externas nos idosos são muito próximos aos da faixa etária de adolescentes e adultos jovens, os quais exibem altos coeficientes e grande número de casos de trauma por causas externas (acidentes e violência). ${ }^{(4-5)}$

Apesar dos dados terem sido coletados em sistema de prontuário eletrônico, destaca-se a falta de padronização no registro da evolução clínica pelas diversas especialidades, gerando subnotificação de informações importantes, o que dificultou o levantamento de alguns dados e possíveis associações causais e de prognóstico com os eventos traumáticos.

Ressalta-se também a característica do hospital estudado. Por ser de alta complexidade e referência em trauma na região, os casos mais graves são encaminhados especificamente para essa unidade de saúde e é esperado que esse perfil de pacientes seja admitido na UTI. Isso pode ter influenciado as associações com o desfecho mortalidade.

\section{- CONCLUSÃO}

Os idosos vítimas de trauma na UTI apresentaram como principal mecanismo de lesão a queda, especialmente, do mesmo nível, seguida por outras causas externas, sobretudo os atropelamentos. Maior gravidade dos casos, com destaque para o trauma crânio-encefálico grave e o politrauma, favoreceu a ocorrência de múltiplas intercorrências durante a internação, com maior prevalência de infecção pulmonar, traqueostomia decorrente de intubação prolongada ou falha de extubação, choque séptico, uso de hemoderivados e insuficiência renal aguda.

Apesar da capacidade diminuída para enfrentar o estresse imposto pelo trauma, o que leva o idoso a um pior prognóstico, muitos dos pacientes sobreviveram ao evento traumático. Entretanto, foi observada alta mortalidade relacionada ao sexo masculino, à ocorrência de outras causas externas, à gravidade do trauma sofrido e às complicações apresentadas, em especial choque séptico de foco pulmonar e necessidade de suporte dialítico.

Observa-se um aumento na produção científica acerca do trauma no idoso, principalmente no que tange à ocorrência de quedas na comunidade e admissão nos serviços de emergência. Entretanto, quando se trata da internação do idoso vítima de trauma, em estado crítico, na terapia intensiva e suas complicações associadas, as evidências ainda são limitadas. Assim, sugere-se a realização de pesquisas 
adicionais referentes à internação na alta complexidade, a fim de assistir melhor o idoso traumatizado, valorizando suas particularidades fisiológicas, para que possa haver melhor enfrentamento ao trauma, melhor prognóstico e maior sobrevida.

\section{REFERÊNCIAS}

1. de Freitas MG, Bonolo PF, de Moraes EN, Machado CJ. Elderly patients attended in emergency health services in Brazil: a study for victims of falls and traffic accidents. Ciênc. saúde coletiva. [Internet] 2015;20(3) [acesso em 09 fev 2018]. Disponível: http://dx.doi.org/10.1590/1413-81232015203.19582014.

2. de Oliveira FMRL, Fernandes MGM, Barbosa KTF, Pereira MA, dos Santos KFO, Nunes TB. Caracterização do trauma em idosos atendidos em serviço de atendimento móvel de urgência. Rev Rene. [Internet] 2013;14(5) [acesso em 17 jan 2015]. Disponível: http://www.periodicos.ufc.br/rene/article/view/3625.

3. Degani GC, Pereira Júnior GA, Rodrigues RAP, Luchesi BM, Marques S. Idosos vítimas de trauma: doenças preexistentes, medicamentos em uso no domicílio e índices de trauma. Rev. bras. enferm. [Internet] 2014;67(5) [acesso em 25 jan 2015]. Disponível: http://dx.doi.org/10.1590/0034-7167.2014670513.

4. Parreira JG, Soldá SC, Perlingeiro JAG, Padovese CC, Karakhanian WZ, Assef JC. Análise comparativa das características do trauma entre pacientes idosos e não idosos. RevAssocMed Bras. [Internet] 2010; 56(5):541-6 [acesso em 14 jan 2015]. Disponível: https://www.researchgate.net/profile/Jose_Parreira/publication/262519018_Comparative_analysis_ of_trauma_characteristics_between_elderly_and_younger_trauma_patients/links/543d091b0cf24ef33b765afc.pdf.

5. Lima RS, Campos MLP. Perfil do idoso vítima de trauma atendido em uma Unidade de Urgência e Emergência. RevEscEnferm USP. [Internet] 2011;45(3) [acesso em 14 jan 2015]. Disponível: http://www.redalyc.org/articulo. oa? id=361033310016.

6. Rodrigues J, Ciosak SI. Idosos vítimas de trauma: análise de fatores de risco. RevEscEnferm USP. [Internet] 2012;46(6) [acesso em 12 jan 2015]. Disponível: http://www.redalyc.org/articulo.oa?id=361033321017.

7. Monteiro CR, Mancussi e Faro AC. Avaliação funcional de idoso vítima de fraturas na hospitalização e no domicílio. RevEscEnferm USP. [Internet] 2010;44(3) [acesso em 25 jan 2015]. Disponível: http://www.redalyc.org/articulo. oa? id=361033305023.

8. Navarro JTM, Estrada AF, López RM, Díaz OLC. Caracterización de adultos mayores hospitalizados con lesiones traumáticas osteomioarticulares. Medisan. [Internet] 2011;15(7) [acesso em 27 jan 2015]. Disponível: http://scielo.sld. cu/scielo.php?script=sci_arttext\&pid=S1029-30192011000700005\&lng=es.

9. Bonne S, Schuerer DJE. Trauma in the Older Adult. ClinGeriatr Med. [Internet] 2013;29(1) [acesso em 12 jan 2015 ]. Disponível: http://dx.doi.org/10.1016/j.cger.2012.10.008.

10. Meschial WC, Soares DFPP, de Oliveira NLB, Nespollo AM, da Silva WA, Santil FLP. Elderly victims of falls seen by prehospital care: gender differences. Rev. bras. epidemiol. [Internet] 2014;17(1) [acesso em 09 fev 2018]. Disponível: http://dx.doi.org/10.1590/1415-790X201400010002ENG.

11. Souza JAG, Iglesias ACRG. Trauma no idoso. Rev. Assoc. Med. Bras. [Internet] 2002;48(1) [acesso em 10 jan 2015 ]. Disponível: http://dx.doi.org/10.1590/S0104-42302002000100037.

12. Fhon JRS, Rosset I, Freitas CP, Silva AO, Santos JLF, Rodrigues RAP. Prevalência de quedas de idosos em situação de fragilidade. Rev Saúde Pública. [Internet] 2013;47(2) [acesso em 17 jan 2015]. Disponível: http://dx.doi.org/10.1590/ rsp.v47i2.76629.

13. Cavalcante ALPM, de Aguiar JBD, Gurgel LA. Fatores associados a quedas em idosos residentes em um bairro de Fortaleza, Ceará. Rev. bras. geriatr. gerontol. [Internet] 2012;15(1) [acesso em 14 jan 2015]. Disponível: http://dx.doi. org/10.1590/S1809-98232012000100015.

14. Broska Júnior CA, de Folchini $A B$, de Ruediger RR. Estudo comparativo entre o trauma em idosos e não idosos atendidos em um Hospital Universitário de Curitiba. Rev. Col. Bras. Cir. [Internet] 2013;40(4) [acesso em 17 jan 2015 ]. Disponível: http://dx.doi.org/10.1590/S0100-69912013000400005. 
15. Fernández BU, Trevigno AB, Luna FG, Fernández FB. Relación entre mortalidad hospitalaria y edad en pacientes con trauma encéfalo craneano moderado y grave. Rev Anacem. [Internet] 2012;6(1) [acesso em 14 jan 2015]. Disponível: http://bases.bireme.br/cgi-bin/wxislind.exe/iah/online/? IsisScript=iah/iah.xis\&src=google\&base=LILACS\&lang=p\&next Action=Ink\&exprSearch=640036\&indexSearch=ID.

16. Patel HC, Bouamra O, Woodford M, Yates DW, Lecky FE. Clinical article: mortality associated with severe head injury in the elderly. Acta Neurochir. [Internet] 2010;152(8) [acesso em 09 fev 2018]. Disponível: http://dx.doi.org/10.1007/ s00701-010-0666-x.

17. Cardoso LT, Grion CM, Matsuo T, Anami EH, Kauss IA, Seko L, et al. Impact of delayed admission to intensive care units on mortality of critically ill patients: a cohort study. CritCare. [Internet] 2011;15(1) [acesso em 13 mar 2018 ]. Disponível: http://dx.doi.org/10.1186/cc9975.

18. Churpek MM, Wendlandt B, Zadravecz FJ, Adhikari R, Winslow C, Edelson DP. Association between intensive care unit transfer delay and hospital mortality: a multicenter investigation. J. Hosp. Med. [Internet] 2016;11(11) [acesso em 13 mar 2018]. Disponível: http://dx.doi.org/10.1002/jhm.2630.

19. Ong AW, Omert LA, Vido D, Goodman BM, Protetch J, Rodriguez A, et al. Characteristics and outcomes of trauma patients with ICU lengths of stay 30 days and greater: a seven-year retrospective study. CriticalCare. [Internet] 2009;13(5) [acesso em 17 jan 2015]. Disponível: https://doi.org/10.1186/cc8054.

20. Frederickson TA, Renner CH, Swegle JR, Sahr SM. The cumulative effect of multiple critical care protocols on length of stay in a geriatric trauma population. J IntensiveCare Med. [Internet] 2013;28(1) [acesso em 17 jan 2015]. Disponível: http://dx.doi.org/ 10.1177/0885066611432420.

21. Costa CDS, Scarpelini S. Avaliação da qualidade do atendimento ao traumatizado através do estudo das mortes em um hospital terciário. Rev. Col. Bras. Cir. [Internet] 2012;39(4) [acesso em 24 jan 2015]. Disponível: http://www. producao.usp.br/handle/BDPI/38669. 